\title{
Activité physique, connaissances en santé et habitudes alimentaires d'élèves belge : effets de la méthodologie Oblomov
}

\author{
Nicolas Franck, Maurine Remacle, Alexandre Mouton et Marc Cloes
}

\author{
Nicolas Franck. \\ Maurine Remacle. \\ Alexandre Mouton. \\ Marc Cloes.
}

Résumé. En Belgique, seuls 2\% des enfants âgés de 6 à 9 ans atteignent les recommandations internationales d'activité physique (AP). Ce constat souligne le besoin d'actions concrètes pour réduire l'inactivité des préadolescents, particulièrement au cours d'éducation physique (EP). Développé dans le cadre d'un programme Erasmus +, le projet ObLoMoV (https://www.oblomovproject.eu) propose une combinaison originale d'imaginaire et de HIIT. L'objectif de cette étude consiste à analyser l'influence de cette approche pédagogique sur l'AP auto-rapportée, les connaissances en santé et les habitudes alimentaires d'élèves de l'enseignement fondamental. Cela s'inscrit dans le cadre du modèle d'analyse du processus d'enseignement-apprentissage. Cinq enseignants en EP ont proposé un cycle de 10 leçons à 176 élèves de $5^{\text {ème }}$ et $6^{\text {ème }}$ années. Chaque leçon combinait High Intensity Interval Training (HIIT), théâtralisation et éducation à la santé.

Mots-clés. Activité physique, Éducation physique, High Intensity Interval Training, Jeu, Enseignement primaire

\section{Introduction}

En Belgique, seuls 2\% des enfants de 6 à 9 ans atteignent les recommandations mondiales en matière d'activité physique (Wijtzes et al., 2016). Étant étendu que les habitudes physiques acquises au plus jeune âge ont tendance à se maintenir à l'âge adulte, ceci souligne la nécessité de promouvoir un style de vie actif auprès de la jeune génération (Morris, 2013). Le cours d'éducation physique est considéré par l'UNESCO comme l'outil le plus pertinent pour transmettre les compétences et connaissances nécessaires à l'acquisition d'un style de vie actif pour la vie (McLennan \& Thompson, 2015). En effet, selon Chin et Edington (2014), chaque leçon d'éducation physique devrait s'inscrire dans une démarche d'éducation à la santé. Chaque activité proposée devrait l'être dans la perspective de son utilisation concrète par l'élève, en vue de l'adoption d'un style de vie actif. Cependant, contrairement à l'avis général des praticiens, Green (2014) constate que l'on ne retrouve aucune preuve dans la littérature que le cours d'éducation physique est ou sera un jour capable d'impacter l'adoption à long terme d'un style de vie actif par les élèves. Pour y parvenir, Cloes (2017) souligne que l'enseignant devrait plus souvent donner du sens et mettre en évidence les liens existants entre les activités qu'il propose et l'utilisation concrète que les élèves pourraient en retirer. En plus du manque de sens des activités, Boudreau (2004) considère que ni les situations de match, ni les activités de type drills, pourtant légions dans l'enseignement de l'éducation physique, ne permettent de susciter chez les élèves l'envie de s'impliquer à long terme dans l'activité physique. Enfin, plus spécifiquement, les activités de conditionnement physique peuvent engendrer des difficultés psycho-physiques qui deviennent alors des barrières importantes à l'activité physique (Vitale, 2018).

C'est à partir de ces constats qu'est née l'approche Oblomov qui combine le HIIT (High Intensity Interval Training) et la contextualisation imaginaire des leçons. Vitale (2018) identifie quatre avantages du HIIT : durées d'effort réduites, participation possible des enfants déconditionnés et en surpoids, similarité avec le caractère intermittent des mouvements naturels chez l'enfant et, enfin, forme d'entrainement bien moins monotone et donc plus amusante. D'un autre côté, la mise en contexte imaginaire des exercices constitue une source de motivation permettant d'inciter les élèves à bouger (Pasetti, 2018). La méthodologie Oblomov tente d'apporter une touche pédagogique originale dans l'enseignement de l'éducation physique en Europe. Sa version belge a été adaptée dans le sens où des animations 
Activité physique, connaissances en santé et habitudes alimentaires d'élèves belge : ef...

d'éducation à la santé ont été introduites lors de chacune des leçons afin d'être en conformité avec les nouveaux défis auxquels la profession fait face à l'heure actuelle (Éducation Physique de Qualité, Pacte pour un Enseignement d'Excellence). Pour favoriser l'impact concret de l'intervention et changer réellement le comportement des élèves en dehors des murs de l'école, lors de chaque leçon, l'enseignant va récolter les bonnes pratiques santé mises en place par les élèves à domicile.

L'objectif principal de cette étude consiste à analyser l'impact sur les élèves de $5^{\text {ème }}$ et $6^{\text {ème }}$ années primaires d'un cycle de leçons d'éducation physique appliquant les principes de l'approche Oblomov.

\section{Méthodologie}

Cette étude répond aux caractéristiques des recherches actions (Willis \& Edwards, 2014). L'objectif consistait à analyser la mise en place de l'innovation pédagogique et à dégager d'éventuelles pistes d'amélioration ainsi que mettre en évidence d'éventuels changements au sein du groupe de sujets.

Le cycle de leçons a été conçu sur une base de 10 séances (une par semaine). Chacune des leçons devait compter 60 minutes effectives de pratique. Elles devaient respecter les principes directeurs du projet à savoir : l'intégration des situations de HIIT, la mise en contexte imaginaire, l'organisation d'animations d'éducation à la santé lors de chaque leçon et la création de scénarios par les élèves à la fin du cycle et leur enregistrement vidéo.

Au total, cinq écoles de la région liégeoise et 176 élèves, répartis dans huit classes différentes, ont pris part à ce projet. Un total de 80 leçons de type Oblomov a donc été dispensé et cinq enseignants en éducation physique ont été formés à cette pédagogie particulière.

Concernant les outils de mesure utilisés, les élèves ont rempli un questionnaire avant (T0) et après (T1) la mise en place du cycle. L'objectif majeur de cet instrument consistait à évaluer le niveau d'activité physique, le fonctionnement émotionnel et les habitudes alimentaires des élèves avant l'intervention. Pour y parvenir, nous avons combiné différents instruments validés. En ce qui concerne l'activité physique, nous avons utilisé le questionnaire PAQ-C (Physical Activity Questionnaire for Older Children, Kowalski, Crocker, \& Donen, 2004). Afin d'évaluer le fonctionnement émotionnel, nous avons soumis les élèves au TEIQue-ASF (Trait Emotional Intelligence Questionnaire - Adolescent Short Form, Petrides, 2009). Les habitudes alimentaires des élèves ont été analysées via l'Adolescent Food Habits Checklist (Johnson, Wardle, \& Griffith, 2002). Par l'intermédiaire d'un quiz, nous avons également évalué les connaissances en matière de santé avant et après l'intervention.

Aussi, à l'issue de chaque séance, les élèves ont rempli un court questionnaire de perception de leçon qui portait sur trois aspects fondamentaux de la qualité d'une leçon d'éducation physique : l'apprentissage, la dépense énergétique et l'amusement des élèves. A partir de la deuxième séance, tout au début de chaque leçon, les élèves ont également décrit les comportements en lien avec les animations santé qui ont été proposées et qu'ils ont eu l'occasion de mettre en œuvre depuis la séance précédente et le début du cycle.

Bien évidemment, les instruments utilisés nous ont permis de récolter des données nécessitant un traitement principalement quantitatif mais certaines variables ont été traitées de manière qualitative.

\section{Résultats et discussion}

\section{Impact du cycle}

A l'issue du cycle, les résultats aux tests de connaissances en matière de santé se sont améliorés dans la majorité 
Actes de la 11ème Biennale de l'ARIS : Former des citoyens physiquement éduqués. Axe 1 - Ecole en santé, 10.25518/ sepaps 20.260

des classes $(3,22$ à 4,37/10; $\mathrm{p}<0,000)$. Nos résultats corroborent ceux de la littérature, confirmant qu'il est possible d'améliorer les connaissances « santé » de l'élève par une intervention de l'enseignant (Sherman \& Muelhoff, 2007). Le fait de proposer des activités d'éducation à la santé de manière hebdomadaire semble porter ses fruits, ce qui souligne l'importance du rôle du professeur d'éducation physique en matière d'éducation à la santé (Turcotte, 2008).

Si nous remarquons une franche évolution au niveau des connaissances, il n'en va pas de même pour les habitudes alimentaires puisque ces dernières ne se sont pas améliorées et ont même quelque peu régressé au cours de l'intervention (de 13,88 à 13,55; p=0,24). Une explication probable est que les habitudes alimentaires des enfants étaient principalement déterminées par les parents et, surtout, par la mère de l'enfant (Veereecken, Keukelier \& Maes, 2004).

En ce qui concerne l'intelligence émotionnelle, il n'y a pas non plus d'amélioration significative des résultats (de 143,41 à 144,$15 ; \mathrm{p}=0,77)$. Cela peut s'expliquer aussi par la relativement courte durée d'intervention.

Enfin, concernant le niveau d'activité physique auto-rapporté (PAQ-C), à l'issue du cycle, il a significativement augmenté $(3,09$ à 3,$26 ; p<0,000)$. Le cycle semble donc avoir permis aux élèves d'améliorer leur niveau d'activité physique hebdomadaire à court-terme. Les niveaux d'activité physique rapportés sont significativement supérieurs chez les garçons par rapport à ceux des filles $(\mathrm{p}=.0048)$. Ces résultats concordent avec la littérature puisque de nombreuses études ont abouti aux mêmes conclusions (Biddle, Gorely \& Stensel, 2004).

Néanmoins, après le cycle, la différence entre les deux sexes est nettement réduite et n'est plus significative. Le niveau d'activité physique s'est donc amélioré davantage chez les filles. Cet attrait des filles pourrait s'expliquer par l'approche non- compétitive et centrée sur la tâche prônée par le cycle Oblomov, qui correspond mieux aux caractéristiques motivationnelles des filles (Roberts \& Walker, 2001).

\section{Avis et réactions des élèves à propos du projet}

A partir de la figure 1 , on peut constater que, dans certaines classes ( $\mathrm{C} 1, \mathrm{C} 4, \mathrm{C} 6)$, les élèves réagissent de façon très positive tandis que dans d'autres, ils semblent moins bien se comporter avec de multiples comportements hors-tâches (C2, C3) (Franck, Mouton, Remacle \& Cloes, 2019). Le taux de participation, excellent dans les classes C5 à C8 et très positif dans les classes $\mathrm{C} 1$ à $\mathrm{C} 4$, souligne un intérêt des élèves pour la méthode (figure 1). Les données recueillies confirment donc les arguments en faveur du HIIT (Reichert, Barros, Domingues \& Hallal, 2007 ; Vitale, 2018). Par rapport au temps d'engagement moteur, la plupart des élèves (76\%) répondent avoir beaucoup bougé et l'item « Mouvement perçu » est l'item qui obtient les scores les plus élevés (Franck et al., 2019). Ceci va à l'encontre des craintes émises par les enseignants avant l'intervention, même si les élèves ont généralement tendance à surestimer leur engagement réel (Metzler, 1989). Enfin, concernant l'apprentissage perçu, seuls $47 \%$ des élèves interrogés ont répondu qu'ils avaient appris quelque chose au cours de la leçon (Franck et al., 2019). C'est étonnant puisque la comparaison des résultats aux tests théoriques entre T0 et T1 montre une amélioration significative des résultats. 
Activité physique, connaissances en santé et habitudes alimentaires d'élèves belge : ef...

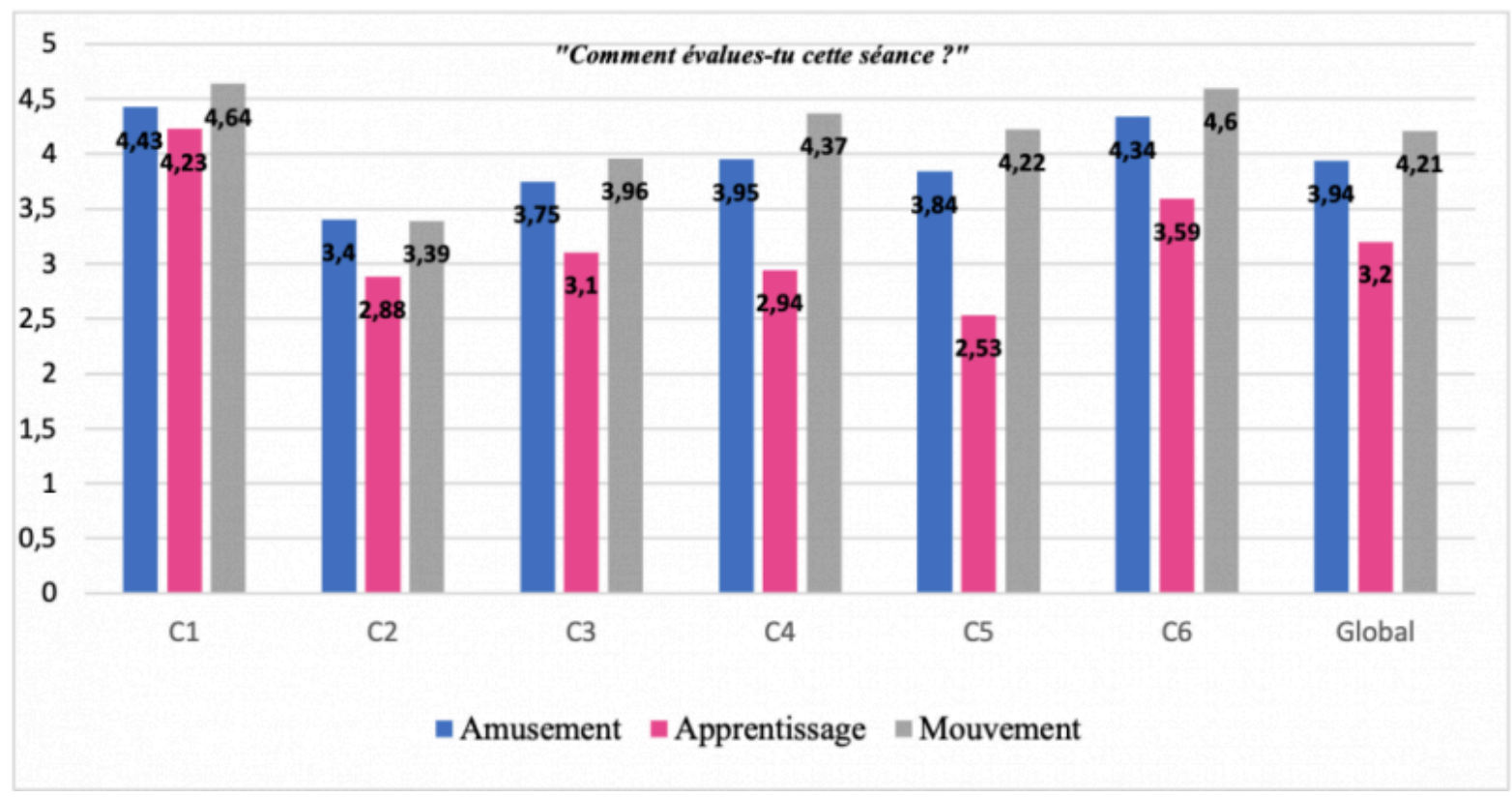

Figure 1 : Perceptions globales du cycle par les élèves (/5)

\section{Limites de l'étude}

Tout d'abord, les participants à cette étude étaient tous volontaires, ce qui conduit à un éventuel biais de sélection. Ensuite, nous avons laissé aux enseignants la possibilité d'adapter le contenu des leçons. La manière avec laquelle le cycle a été dispensé n'a donc pas été identique d'une école à l'autre voire d'une classe à l'autre, ce qui implique d'en tenir compte au moment de comparer les résultats. Rappelons aussi que les données récoltées correspondent aux déclarations des élèves. Nous n'avons pas de preuves formelles de leur mise en place.

\section{Conclusion}

L'objectif principal de cette étude était d'analyser l'impact sur les élèves d'un cycle de leçons d'éducation physique appliquant les principes de l'approche Oblomov en 5ème et 6ème années primaires. Les élèves ont réagi positivement à l'implémentation de cette approche pédagogique originale et ont manifestement apprécié les activités proposées. Au vu des résultats présentés, le cycle Oblomov a permis aux élèves d'acquérir des connaissances théoriques en matière de santé ainsi que d'accroitre leur niveau de pratique d'activité physique déclaré. Soulignons aussi que l'adaptation belge a permis aux élèves de proposer de nombreuses bonnes pratiques santé et d'envisager des adaptations concrètes de leur style de vie. Néanmoins, pour réellement ancrer ces changements et pérenniser les bénéfices, l'implication des parents au sein du projet apparaît indispensable. Les constats présentés soulignent la pertinence de l'approche Oblomov dans l'enseignement primaire. Par conséquent, il serait judicieux d'attirer l'attention des responsables de l'éducation afin d'intégrer ce type d'activités au programme du cours d'éducation physique et, pourquoi pas, à la formation initiale et continue des professeurs d'éducation physique.

\section{Bibliographie}

Biddle, S., Gorely, T., \& Stensel, D. (2004). Health-enhancing physical activity and sedentary behaviour in children and adolescents. Journal of Sports Sciences, 22(8), 679-701. http://doi.org/10.1080/02640410410001712412 
Actes de la 11ème Biennale de l'ARIS : Former des citoyens physiquement éduqués. Axe 1 - Ecole en santé, 10.25518/ sepaps 20.260

Boudreau, P. (2004). Développer le goût de l'activité physique et l'enseignement de l'éducation physique à l'école secondaire: un rendez-vous manqué. Avante, 10(2), 17-23.

Chin, M.-K., \& Edginton, C.R. (2014). Physical Education and Health Global Perspectives and Best Practice. Urbana, IL: Sagamore.

Cloes, M. (2017). Preparing physically educated citizens in physical education. Expectations and practices. Retos, 31, 245-251. Disponible sur http://recyt.fecyt.es/index.php/retos/article/view/53497/32304

Franck, N., Mouton, A., Remacle, M., \& Cloes, M. (2019 juin). Physical activity, emotional intelligence and food habits of Belgian pupils: effects of the Oblomov methodology. Communication présentée lors de la 2019 AIESEP International Conference: Building Bridges for Physical Activity and Sport. Garden City, NY: Adelphi University. Disponible sur http://hdl.handle.net/2268/240972

Green, K. (2014). Mission impossible? Reflecting upon the relationship between physical education, youth sport and lifelong participation. Sport, Education and Society, 19(4), 357- 375.

Doi : $10.1080 / 13573322.2012 .683781$.

Johnson, F., Wardle, J., \& Griffith, J. (2012). The adolescent food habits checklist: reliability and validity of a measure of healthy eating behaviour in adolescents. European Journal of Clinical Nutrition, 56(7), 644-649. http://doi.org/10.1038/sj.ejcn.1601371

Kowalski, K, Crocker, P., \& Donen, R. (2004). The physical activity questionnaire for older children (PAQ-C) and adolescents (PAQ-A) manual.

McLennan, N., \& Thompson, J. (2015). Quality Physical Education (QPE). Guidelines for policy-makers. Paris, France: UNESCO.

Metzler, M. (1989). A review of research on time in sport pedagogy. Journal of Teaching in Physical Education, 8(2), 87-103.

Morris, C. (2013). Designed to Move: Tackling the Epidemic of Physical Inactivity. National Civic Review, 102(4), 55-56. http://doi.org/10.1002/ncr.21157

Pasetti, L. (2018). Theater. Paper presented at the 2018 AIESEP World Congress 'Creating thriving and sustainable futures'. University of Edinburgh, Edinburgh, United Kingdom.

Petrides, K. (2009). Psychometric properties of the Trait Emotional Intelligence Questionnaire (TEIQue). In C. Stough, D. H. Saklofske, \& J. D. A. Parker (Eds.), The Springer series on human exceptionality. Assessing emotional intelligence: Theory, research, and applications (pp 85-101). New York, NY, US: Springer Science + Business Media. http://doi.org/10.1007/978-0-387-88370-0 5

Reichert, F.F., Barros, A.J., Domingues, M.R., \& Hallal, P.C. (2007). The role of perceived personal barriers to engagement in leisure-time physical activity. American Journal of Public Health, 97, 515-519.

Roberts, G., \& Walker, B. (2001). La théorie des buts d'accomplissement dans le domaine du sport et des activités physiques. In F. Cury,, \& P. Sarrazin (Eds.), Théories de la motivation et pratiques sportives : état des recherches (pp. 97-120). Paris, France: Presses Universitaires de France.

Sherman, J., \& Muehlhoff, E. (2007). Developing a Nutrition and Health Education Program for Primary Schools in 
Activité physique, connaissances en santé et habitudes alimentaires d'élèves belge : ef...

Zambia. Journal of Nutrition Education and Behavior, 39, 335-342.

Turcotte, S. (2008). L'inclusion de l'éducation à la santé en éducation physique : analyse des pratiques pédagogiques d'éducateurs physiques du primaire. Revue des sciences de l'éducation, 34(2), 512-513. http://doi.org/10.7202/ $\underline{019710 \mathrm{ar}}$

Vereecken, C., Keukelier, E. \& Maes, L. (2004). Influence of mother's educational level on food parenting practices and food habits of young children. Appetite, 43, 93-103.

Vitale, J. (2018). Integrating high intensity intermittent training (HIIT) in theatre open scenarios: foundations of an original approach. Paper presented at the 2018 AIESEP World Congress 'Creating thriving and sustainable futures'. University of Edinburgh, Edinburgh, United Kingdom.

Wijtzes, A.I., Verloigne, M., Mouton, A., Cloes, M., De Ridder, K.A.A., Cardon, G., \& Seghers, J. (2016). Results from the 2016 Active Healthy Kids Belgium Report Card on Physical Activity for Children and Youth. Journal of Physical Activity and Health, 13(2), 95-103. http://doi.org/10.1123/jpah.2016-0306

Willis, J., \& Edwards, C. (2014). Action Research Models, Methods and Examples. Charlotte, NC: Information Ages Publishing.

PDF automatiquement généré le 2023-04-26 04:52:15

Url de l'article : https://popups.uliege.be/sepaps20/index.php?id=260

Publié par ULiège Library en Open Access et distribué suivant les termes et les conditions de la licence CC-BY (https://creativecommons.org/licenses/by/4.0/deed.fr) 